\title{
Posttraumatic growth and well-being among adolescents and young adults (AYAs) with cancer: a longitudinal study
}

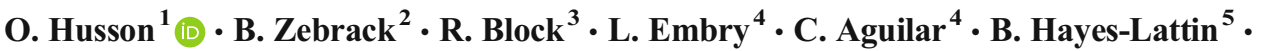 \\ S. Cole ${ }^{6}$
}

Received: 19 October 2016 / Accepted: 10 April 2017 / Published online: 19 April 2017

(C) The Author(s) 2017. This article is an open access publication

\begin{abstract}
Purpose This study examines posttraumatic growth (PTG) among adolescents and young adults (AYAs) with cancer, as well as its correlates and trajectories over time. The study also explores the buffering role of PTG on the associations between posttraumatic stress (PTS), health-related quality of life (HRQoL), and psychological distress.

Methods A multicenter, longitudinal, prospective study was conducted among AYA cancer patients aged 14-39 years. One hundred sixty-nine patients completed a self-report measure of PTG (PTGI) and PTS (PDS) 6, 12, and 24 months after baseline (within the first 4 months of diagnosis). At 24-month follow-up, HRQoL (SF-36) and psychological distress (BSI18) were also assessed.

Results Among participants, 14\% showed increasing PTG, $45 \%$ remained at a stable high PTG level, $14 \%$ showed decreasing PTG, and 27\% remained at a stable low PTG level. AYAs who remained high on PTG were more often younger, female, and received chemotherapy. PTG level at 6-month follow-up was predictive of mental HRQoL ( $\beta=0.19$; $p=0.026)$ and psychological distress $(\beta=-0.14 ; p=0.043)$
\end{abstract}

O. Husson

Olga.Husson@radboudumc.nl

1 Department of Medical Psychology, Radboud University Medical Center, PO Box 9101, 6500, HB Nijmegen, The Netherlands

2 University of Michigan School of Social Work, Ann Arbor, MI, USA

MNR Analytics, Portland, OR, USA

4 Pediatric Hematology/Oncology, University of Texas Health Science Center at San Antonio, San Antonio, TX, USA

5 Oregon Health and Sciences University, Portland, OR, USA

6 HopeLab Foundation, Redwood City, CA, USA at 24-month follow-up when corrected for PTS and sociodemographic and clinical covariates. No relationship between PTG and physical HRQoL was found. The interactive effects of PTS and PTG on outcomes were not significant, indicating that buffering did not take place.

Conclusion This study indicates that PTG is dynamic and predicts mental well-being outcomes but does not buffer the effects of PTS. Psychosocial interventions should focus on promoting PTG and reducing PTS in order to promote the adjustment of AYAs diagnosed with cancer.

Keywords Adolescent and young adult · Posttraumatic growth $\cdot$ Health-related quality of life $\cdot$ Posttraumatic stress · Psychological distress · Oncology

\section{Introduction}

The common trend of combining the adolescents and young adults (AYA) with cancer, defined here as persons diagnosed between 15 and 39 years of age at time of diagnosis [1], with either pediatric or older adult cancer populations in psychosocial research has created a gap in understanding the cancer experience of AYAs [2]. Adolescence and young adulthood are life stages characterized by increased vulnerability to stress as age-related developmental tasks need to be achieved [3, 4], such as completing school and becoming independent from parents for adolescents and starting a family and getting a mortgage for young adults. A cancer diagnosis challenges AYAs' abilities to achieve these developmental milestones [5] and can compromise AYAs' adjustment and healthrelated quality of life (HRQoL) [6].

Much of today's psychosocial oncology research harkens to Selye's theoretical stress and coping models, in which human emotional response to upsetting or traumatic events or 
conditions may be experienced as "distress" (negative) or "eustress" (positive) [7]. Subsequent applications of stress coping theory to investigations of patients' psychosocial adaptation to cancer suggest that it is theoretically possible to experience both positive and negative responses to cancer simultaneously. Tedeschi and Calhoun have conceptualized positive adaptation to traumatic life events, including serious illness, as posttraumatic growth (PTG) [8,9]. Positive changes reported by some cancer survivors have included a greater sense of closeness to others, higher appreciation of everyday life, recognition of new possibilities in life, a sense of personal strength, and deeper spirituality [10]. In a semi-structured interview study of AYAs' diagnosed and treated for cancer [11], two recurrent themes emerged: (1) loss of control resulting in a sense of frustration and anger and (2) benefit finding such as improved personal attributes and strengthened relationships. All but one participant reported that they experienced at least one positive aspect of being treated for cancer. Quantitative research examining the prevalence of PTG in AYA cancer populations is scarce but suggests that most AYA cancer patients report some PTG [12-16].

There is controversy in the literature about how to conceptualize PTG: as an outcome of coping with a stressful life event similar to well-being [17] or as a process of coping with adversity, which may lead to positive adjustment [18] but is conceptually distinct from it. Findings regarding the relationships between PTG and well-being in cancer patients have been inconsistent; some studies have found positive (curvilinear) correlations with adjustment outcomes, while others find negative relations or even null findings [10, 19]. Several hypotheses may explain these inconsistencies including varying PTG levels over time within individuals. However, another possible explanation for these inconsistencies is that PTG may influence well-being not only as a direct effect but also through a stress-buffering mechanism. The ability to identify positive aspects of their cancer experience may help to protect patients from the adverse effects of cancer and its treatment. For example, a study of breast cancer patients showed that PTG moderated relationships between posttraumatic stress (PTS) and both psychological distress and HRQoL [20]. These authors suggested that PTG may help patients to reinterpret and accept the threatening aspects of the experience, thus restoring a sense of security and lessening the impact of PTS symptoms on well-being.

Prior investigations indicated that $39-48 \%$ of AYA cancer patients report PTS symptoms [21, 22], and that these symptoms negatively impact HRQoL and psychological distress [23]. It is therefore important to know if PTG is simply an outcome or if it can also act as a stress-buffering process. Considering the gaps in our understanding of PTG among AYAs with cancer, the aims of this study are to (1) examine trajectories of PTG over time, (2) identify the correlates that distinguish PTG trajectories, (3) determine the predictive value of PTG on HRQoL and psychological distress, and (4) examine the buffering role of PTG on the association between PTS and HRQoL and psychological distress. Findings will help health care providers readily identify AYAs who may benefit from targeted interventions that not only aim to reduce psychological distress but also promote positive adaptation and growth.

\section{Methods}

\section{Design, procedure, and patients}

A prospective, longitudinal, multisite study was conducted to examine HRQoL outcomes over 2 years in AYA patients recently diagnosed with cancer. Baseline data were collected within the first 4 months of diagnosis and subsequently at 6 , 12 , and 24 months after the baseline survey. The current study focuses on assessments of PTG administered at the three follow-up points. Eligibility criteria included patients aged 14 (and anticipated to turn 15 years old during treatment) through 39 years, with a first diagnosis of any form of invasive cancer, and the ability to read and understand English or Spanish. Participating institutions included three pediatric care institutions and two university-affiliated adult care medical institutions. Research staff at each institution monitored clinic rosters and subsequently identified and approached a total of 286 eligible patients over a 25 -month period. Fifty-eight patients did not provide consent, either because they refused participation or because physicians denied access to patients who were too sick to participate. An additional 12 AYAs did not return a completed survey after providing consent, and one died before completing the baseline survey. Overall participation rate was $75 \%(n=215)$. Institutional Review Board approval was obtained from each participating site and coordinating center. Informed consent and/or assent was obtained from patients and parents.

\section{Measures}

Posttraumatic growth was measured by the Posttraumatic Growth Inventory (PTGI) [24] at 6- (T1), 12- (T2), and 24month (T3) follow-up. Twenty-one items were assessed on a 6-point ordinal scale ranging from 0 (no change at all) to 5 (very great change) for 5 subscales: new possibilities ( 5 items), relating to others ( 7 items), personal strength (4 items), spiritual change (2 items), and appreciation of life (3 items). Cancer diagnosis was stated in the question stem as the reference for endorsing items. Scores for each of the five subscales were derived by summing response values, and all items were totaled to determine an overall summated PTGI score, ranging from 0 to 105 . Higher scores indicated greater growth. We also computed the proportions of participants endorsing single 
items at a moderate or great level (item score $\geq 3$ ) to be able to characterize the most common and frequent forms of PTG [25] and used a cut-off of $\geq 63$ to classify low vs. high PTG levels as was done in previous studies [26-28]. Cronbach's alpha ranged from .84 to .91 for the 5 subscales of the PTGI. Cronbach's $\alpha$ was 0.95 for the overall score.

Social support was measured at baseline (T0) with the Medical Outcomes Survey Social Support survey, which is a 19-item questionnaire assessing the degree to which a chronically ill patient perceives availability of functional social support [29].

Posttraumatic stress symptoms were assessed at 6-, 12, and 24-month follow-up using the Posttraumatic Stress Diagnostic Scale (PDS) [30]. This measure includes 17 questions covering three categories of symptoms: re-experiencing ( 5 items), avoidance ( 7 items), and arousal ( 5 items). We adapted the items in the PDS by replacing the word "trauma" with "cancer" in order to specifically investigate cancer-related PTSS. Item scores are summed to derive an overall posttraumatic stress severity score ranging from 0 to 51 .

Health-related quality of life (HRQoL) was measured at 24month follow-up (T3) by the Medical Outcomes Study Short Form-36 Health Survey (SF-36) [31]. Two higher order component scores were calculated, one for physical health (Physical Component Score (PCS)) and one for mental health (Mental Component Score (MCS)). Based on 1998 US population data, raw scores of MCS and PCS are transformed into $T$ scores ranging from 0 to 100 , with higher scores representing better HRQoL.

Psychological distress was assessed at 24-month (T3) follow-up with the Brief Symptom Inventory-18 (BSI-18), which contains 18 self-report items [32] and comprises a Global Symptom Index (GSI). Raw scores for the GSI were converted to age- and sex-adjusted $T$ scores for comparison to non-patient community norms (mean, 50; standard deviation, 10). Higher scores indicate greater distress.

Sociodemographic information, including age, sex, race, employment/school status, and relationship/marital status, was reported by patients. Clinical data obtained from medical charts included type of cancer, types of treatment (chemotherapy, radiation, and surgery), and treatment status (on versus off treatment). Surveillance, Epidemiology, and End Results (SEER) codes were used to categorize cancer type into severity of disease [33]. In addition, via self-report at baseline and 24-month follow-up, a symptom checklist, derived by the investigators, elicited subject endorsement of 11 common treatment-related side effects during the past month (i.e., shortness of breath; problems with memory, attention, or concentration; frequent or severe stomach pain, pain in your chest (heartburn), or indigestion; ringing in the ears; pain in your joints; weight loss; frequent fevers; lack of sleep or trouble sleeping; frequent tiredness or fatigue; frequent mouth sores that impact eating and drinking; frequent headaches), which were summed and considered in statistical analyses.

\section{Statistical analyses}

All statistical analyses were performed using SPSS software, version 22.0 (SPSS Inc., Chicago IL), and two-sided $p$ values of $<0.05$ were considered statistically significant.

Differences in sociodemographic and clinical characteristics between patients who completed at least two follow-up questionnaires and those who did not were compared with chisquare or ANOVA, where appropriate. Patients who did not complete two follow-up questionnaires were excluded from further analyses.

Descriptive statistics were used to describe the mean PTG overall and subscale scores. Mean differences over time in PTG total and subscale scores were analyzed with pairedsample $t$ tests. Thereafter, each respondent was assigned to a PTG trajectory group on the basis of PTGI total scores at each of three time points (6-, 12, and 24-month follow-up). AYAs whose PTG scores were $\geq 63$ [26-28] at all three time points were assigned to the high PTG group. Those whose scores exceeded the threshold only at 24-month follow-up, or at 12- and 24-month follow-up, were assigned to the increasing PTG group. Those whose scores exceeded threshold only at 6month follow-up, or at 6- and 12-month follow-up, were assigned to the decreasing PTG group. AYAs whose scores never reached the threshold were assigned to the low PTG group. Six patients had missing values at 12- or 24-month follow-up and were excluded from analysis.

To examine differences in sociodemographic, clinical, and psychosocial characteristics between the four groups, chisquare or ANOVA was used, where appropriate.

To determine the predictive and buffering value of PTG, three multiple hierarchical linear regression equations were estimated, one for each outcome (mental HRQoL, physical HRQoL, psychological distress), in four separate steps: (1) the control variables previously correlated at a significant level $(p<0.10)$ with the outcome measures, (2) adding PTS (mean centered), (3) adding PTG (mean centered), and (4) adding the interaction term PTS $\times$ PTG.

\section{Results}

\section{Characteristics of the study population}

The demographic and clinical characteristics of 169 participants who completed at least two follow-up surveys are summarized in Table 1. Of the 46 non-respondents, 28 were deceased $(60.9 \%)$. Respondents were younger, more often received chemotherapy, and were on treatment at baseline compared to non-respondents and deceased patients. Nonrespondents had a better overall survival expectation (based on 5-year survival data for each specific cancer type) compared to respondents. 
Table 1 Sample characteristics

\begin{tabular}{|c|c|c|c|c|}
\hline & $\begin{array}{l}\text { Respondents with at least } 2 \\
\text { follow-up questionnaires completed } \\
N=169(78.6 \%)^{\mathrm{a}}\end{array}$ & $\begin{array}{l}\text { Non-respondents at follow-up } \\
N=18(8.4 \%)\end{array}$ & $\begin{array}{l}\text { Deceased at follow-up } \\
N=28(13.0 \%)\end{array}$ & $p$ value \\
\hline Sex & & & & 0.09 \\
\hline Female & $77(45.6 \%)$ & $6(33.3 \%)$ & $18(64.3 \%)$ & \\
\hline Male & $92(54.4 \%)$ & $12(66.7 \%)$ & $10(35.7 \%)$ & \\
\hline Race & & & & 0.58 \\
\hline White & $77(46.1 \%)$ & $7(38.9 \%)$ & $11(39.3 \%)$ & \\
\hline Hispanic/Latino & $72(43.1 \%)$ & $8(44.4 \%)$ & $11(39.3 \%)$ & \\
\hline Other & $18(10.8 \%)$ & $3(16.7 \%$ & $6(21.4 \%)$ & \\
\hline Relationship status $^{\mathrm{b}}$ & & & & 0.13 \\
\hline No & $108(65.1 \%)$ & $8(44.4 \%)$ & $14(51.9 \%)$ & \\
\hline Yes & $58(34.9 \%)$ & $10(55.6 \%)$ & $13(48.1 \%)$ & \\
\hline Educational level & & & & 0.06 \\
\hline Less than high school & $86(51.5 \%)$ & $6(33.3 \%)$ & $10(35.7 \%)$ & \\
\hline High school graduate & $22(13.2 \%)$ & $4(22.2 \%)$ & $5(17.9 \%)$ & \\
\hline Some college & $34(20.4 \%)$ & $7(38.9 \%)$ & $4(14.3 \%)$ & \\
\hline College graduate or above & $25(15.0 \%)$ & $1(5.6 \%)$ & $9(32.1 \%)$ & \\
\hline Employment/school status & & & & 0.90 \\
\hline Not occupied ${ }^{\mathrm{c}}$ & $98(59.0 \%)$ & $10(55.6 \%)$ & $15(62.5 \%)$ & \\
\hline Occupied $^{\mathrm{d}}$ & $68(41.0 \%)$ & $8(44.4 \%)$ & $9(37.5 \%)$ & \\
\hline Age at diagnosis, years & & & & 0.012 \\
\hline Mean (SD) & & & & \\
\hline $14-17$ & $79(46.7 \%)$ & $4(22.2 \%)$ & $5(17.9 \%)$ & \\
\hline $18-25$ & $33(19.5 \%)$ & $3(16.7 \%)$ & $7(25.0 \%)$ & \\
\hline $26-39$ & $57(33.7 \%)$ & $11(61.1 \%)$ & $16(57.1 \%)$ & \\
\hline Type of cancer & & & & 0.003 \\
\hline Breast carcinoma & $15(8.9 \%)$ & $1(5.6 \%)$ & $3(10.7 \%)$ & \\
\hline Hodgkin lymphoma & $20(11.8 \%)$ & $3(16.7 \%)$ & $0(0 \%)$ & \\
\hline Non-Hodgkin lymphoma & $13(7.7 \%)$ & $2(11.1 \%)$ & $2(7.1 \%)$ & \\
\hline Testicular carcinoma & $8(4.7 \%)$ & $5(27.8 \%)$ & $1(3.6 \%)$ & \\
\hline Female reproductive & $3(1.8 \%)$ & $2(11.1 \%)$ & $3(10.7 \%)$ & \\
\hline Soft tissue sarcoma & $19(11.2 \%)$ & $0(0 \%)$ & $2(7.1 \%)$ & \\
\hline Brain & $13(7.7 \%)$ & $0(0 \%)$ & $1(3.6 \%)$ & \\
\hline Leukemia & $44(26.0 \%)$ & $1(5.6 \%)$ & $5(17.9 \%)$ & \\
\hline Bone tumors & $21(12.4 \%)$ & $1(5.6 \%)$ & $4(14.3 \%)$ & \\
\hline Other carcinomas ${ }^{\mathrm{e}}$ & $13(7.7 \%)$ & $3(16.7 \%)$ & $7(25.0 \%)$ & \\
\hline Severity of cancer by survival rate & & & & 0.03 \\
\hline$<50 \%$ & $53(31.4 \%)$ & $1(5.6 \%)$ & $9(32.1 \%)$ & \\
\hline $50-80 \%$ & $68(40.2 \%)$ & $6(33.3 \%)$ & $13(46.4 \%)$ & \\
\hline$>80 \%$ & $48(28.4 \%)$ & $11(61.1 \%)$ & $6(21.4 \%)$ & \\
\hline \multicolumn{5}{|l|}{ Type of treatment } \\
\hline Receiving chemotherapy & $147(87.0 \%)$ & $9(52.9 \%)$ & $19(67.9 \%)$ & $<0.001$ \\
\hline Receiving radiation & $29(17.2 \%)$ & $5(29.4 \%)$ & $3(10.7 \%)$ & 0.27 \\
\hline Surgical treatment & $51(30.2 \%)$ & $4(23.5 \%)$ & $11(39.3 \%)$ & 0.50 \\
\hline Treatment status baseline & & & & 0.017 \\
\hline Off treatment & $9(5.3 \%)$ & $4(22.2 \%)$ & $4(14.3 \%)$ & \\
\hline On treatment & $160(94.7 \%)$ & $14(77.8 \%)$ & $24(85.7 \%)$ & \\
\hline
\end{tabular}

${ }^{\mathrm{a}}$ For some variables, the sum of number is not 169 because of missing data

${ }^{b}$ Question was worded as follows: "Are you currently married, living together, or in a significant committed relationship?"

${ }^{\mathrm{c}}$ Response categories included the following: on temporary medical leave/disability, unemployed, and permanently unable to work

${ }^{\mathrm{d}}$ Response categories included the following: employed full time, employed part time, full-time homemaker, and full-time student

${ }^{\mathrm{e}}$ Other carcinomas included thyroid, melanoma, adrenocortical carcinoma, hepatic carcinoma, colorectal carcinoma, head and neck cancer, and other sarcoma

\section{Posttraumatic growth}

The average PTG total and subscale scores at each follow-up point are shown in Fig. 1. At T1, 59\% scored high on PTG, while $64 \%$ at $\mathrm{T} 2$ and $58 \%$ at $\mathrm{T} 3$ scored $\geq 63$. At 6-month follow-up, all AYAs reported at least one form of PTG, while at 12- and 24-month follow-up all but one AYA reported some form of PTG. The most frequently endorsed items were "An appreciation for the value of my own life" (83-87\%), "Knowing I can handle difficulties" (77-81\%), "Knowing that I can count on people in times of trouble" (72-86\%), "Appreciate 


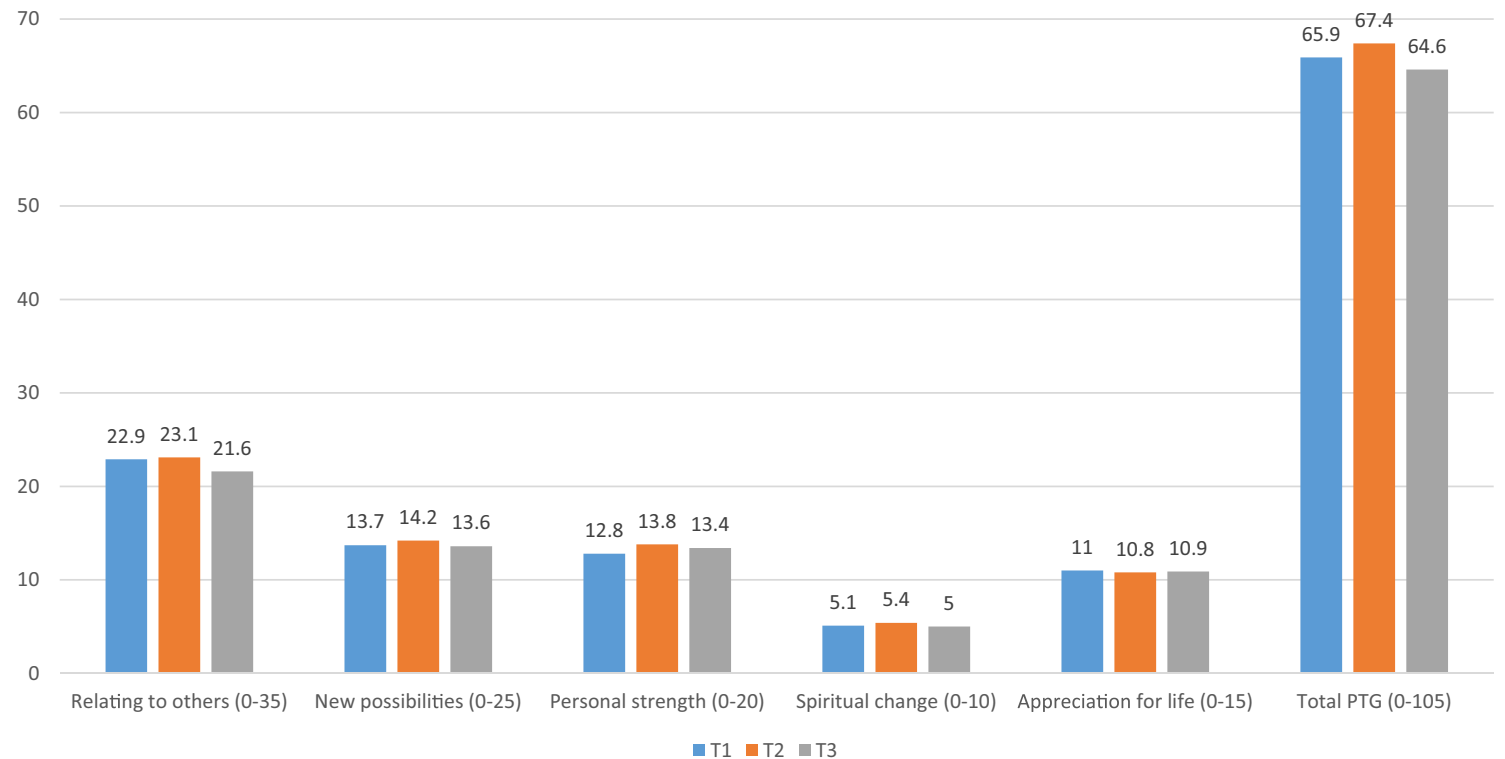

Fig. 1 Mean PTG subscale scores at each time point. T1 6-month follow-up, T2 12-month follow-up, T3 24-month follow-up

each day" (78-80\%), "I discovered that I'm stronger than I thought I was" (69-81\%), "I learned a great deal about how wonderful people are" (70-80\%), and "My priorities about what is important in life" (71-73\%). Paired-sample $t$ tests showed no significant changes in PTG total scores or subscales over time but identified two subscales showing significant change: spiritual change (e.g., "I have a stronger religious faith") which increased from 6 to 12 months $(p=0.006)$ and personal strength (e.g., "I discovered that I am stronger than I thought I was") which improved from 6 to 12 months $(p=0.001)$ and 6 to 24 months $(p=0.003)$.

Among participants, 73 (45\%) had high levels of PTG at all three time points $(84,83$, and $86 \%$, respectively), 23 (14\%) had decreasing PTG levels (70, 64, and 42\%, respectively), 23 (14\%) had increasing PTG levels $(48,67$, and $80 \%$, respectively), and $44(27 \%)$ had low PTG levels at all time points (38, 42, and 34\%, respectively; Fig. 2).

\section{Correlates}

Participants who had high scores on PTG at each time point were more often female, younger, and receiving chemotherapy compared to the other three groups (low PTG, increasing PTG, decreasing PTG; Table 2). No differences between the groups were found in other sociodemographic and cancer-related factors.
Fig. 2 PTG trajectories over time. $T 1$ 6-month follow-up, $T 2$ 12-month follow-up, T3 24month follow-up

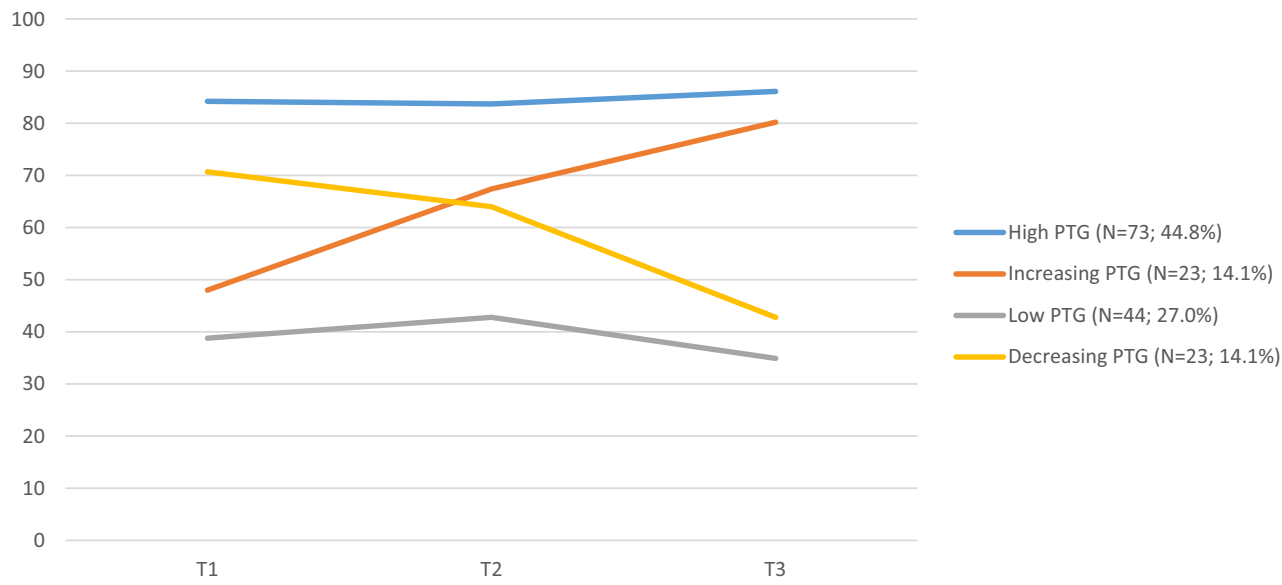


Table 2 Demographic, clinical, and psychosocial characteristics of patients according to their PTG levels over time

\begin{tabular}{|c|c|c|c|c|c|}
\hline & $\begin{array}{l}\text { High PTG } \\
N=73(44.8 \%)\end{array}$ & $\begin{array}{l}\text { Increasing PTG } \\
N=23(14.1 \%)\end{array}$ & $\begin{array}{l}\text { Low PTG } \\
N=44(27.0 \%)\end{array}$ & $\begin{array}{l}\text { Decreasing PTG } \\
N=23(14.1 \%)\end{array}$ & $p$ value \\
\hline \multicolumn{6}{|l|}{ Sociodemographic and clinical characteristics } \\
\hline Sex & & & & & 0.011 \\
\hline Female & $43(58.9 \%)$ & $9(39.1 \%)$ & $18(40.9 \%)$ & $5(21.7 \%)$ & \\
\hline Male & $30(41.1 \%)$ & $14(60.9 \%)$ & $26(59.1 \%)$ & $18(78.3 \%)$ & \\
\hline Race & & & & & 0.82 \\
\hline White & $31(43.1 \%)$ & $10(43.5 \%)$ & $21(47.7 \%)$ & $13(59.1 \%)$ & \\
\hline Hispanic/Latino & $33(45.8 \%)$ & $11(47.8 \%)$ & $17(38.6 \%)$ & $8(36.4 \%)$ & \\
\hline Other & $8(11.1 \%)$ & $2(8.7 \%)$ & $6(13.6 \%)$ & $1(4.5 \%)$ & \\
\hline Relationship status over time & & & & & 0.29 \\
\hline Relationship at all time points & $16(21.9 \%)$ & $5(21.7 \%)$ & $7(15.9 \%)$ & $8(36.4 \%)$ & \\
\hline No relationship at any time point & $38(52.1 \%)$ & $13(56.5 \%)$ & $29(65.9 \%)$ & $7(31.8 \%)$ & \\
\hline Fluctuating & $19(26.0 \% 0$ & $5(21.7 \%)$ & $8(18.2 \%)$ & $7(31.8 \%)$ & \\
\hline Educational level 24-month follow-up & & & & & 0.064 \\
\hline Less than high school & $28(51.9 \%)$ & $6(31.6 \%)$ & $10(26.3 \%)$ & $3(17.6 \%)$ & \\
\hline High school graduate & $10(18.5 \%)$ & $5(26.3 \%)$ & $8(21.1 \%)$ & $3(17.6 \%)$ & \\
\hline Some college & $8(14.8 \%)$ & $4(21.1 \%)$ & $11(28.9 \%)$ & $9(52.9 \%)$ & \\
\hline College graduate or above & $8(14.8 \%)$ & $4(21.1 \%)$ & $9(23.7 \%)$ & $2(11.8 \%)$ & \\
\hline Occupational status over time & & & & & 0.67 \\
\hline Job/school at all time points & $34(47.2 \%)$ & $8(34.8 \%)$ & $17(38.6 \%)$ & $7(30.4 \%)$ & \\
\hline No job/school at any time point & $15(20.8 \%)$ & $7(30.4 \%)$ & $8(18.2 \%)$ & $6(26.1 \%)$ & \\
\hline Fluctuating & $23(31.9 \%)$ & $8(34.8 \%)$ & $19(43.2 \%)$ & $10(43.5 \%)$ & \\
\hline \multicolumn{6}{|l|}{ Age at diagnosis, years } \\
\hline Mean (SD) & $21.9(8.9)$ & $23.7(9.0)$ & $22.2(8.6)$ & $27.7(8.8)$ & 0.046 \\
\hline $14-17$ & $41(56.2 \%)$ & $10(43.5 \%)$ & $21(47.7 \%)$ & $3(13.0 \%)$ & 0.009 \\
\hline $18-25$ & $8(11.0 \%)$ & $4(17.4 \%)$ & $11(25.0 \%)$ & $9(39.1 \%)$ & \\
\hline $26-39$ & $24(32.9 \%)$ & $9(39.1 \%)$ & $12(27.3 \%)$ & $11(47.8 \%)$ & \\
\hline Severity of cancer by survival rate & & & & & 0.78 \\
\hline$<50 \%$ & $24(32.9 \%)$ & $8(34.8 \%)$ & $16(36.4 \%)$ & $4(17.4 \%)$ & \\
\hline $50-80 \%$ & $27(37.0 \%)$ & $9(39.1 \%)$ & $17(38.6 \%)$ & $10(43.5 \%)$ & \\
\hline$>80 \%$ & $22(30.1 \%)$ & $6(26.1 \%)$ & $11(25.0 \%)$ & $9(39.1 \%)$ & \\
\hline Type of treatment (cumulative) & & & & & 0.034 \\
\hline Receiving chemotherapy & $70(100 \%)$ & $21(91.3 \%)$ & $39(88.6 \%)$ & $20(87.0 \%)$ & 0.82 \\
\hline Receiving radiation & $16(26.2 \%)$ & $6(28.6 \%)$ & $8(20.0 \%)$ & $6(35.3 \%)$ & 0.58 \\
\hline Surgical treatment & $44(65.7 \%)$ & $15(75.0 \%)$ & $26(63.4 \%)$ & $17(85.0 \%)$ & \\
\hline Treatment status over time & & & & & 0.29 \\
\hline On treatment at all time points & $16(21.9 \%)$ & $5(21.7 \%)$ & $7(15.0 \%)$ & $8(36.4 \%)$ & \\
\hline Off treatment at all time points & $38(52.1 \%)$ & $13(56.5 \%)$ & $29(65.9 \%)$ & $7(31.8 \%)$ & \\
\hline Fluctuating & $19(26.0 \%)$ & $5(21.7 \%)$ & $8(18.2 \%)$ & $7(31.8 \%)$ & \\
\hline Number of symptoms baseline & & & & & 0.34 \\
\hline Mean & $5.5(2.4)$ & $4.6(3.0)$ & $4.9(2.4)$ & $4.8(2.8)$ & \\
\hline Number of symptoms 24-month follow-up & & & & & 0.81 \\
\hline Mean (SD) & $3.0(2.9)$ & $2.9(2.3)$ & $3.4(2.7)$ & $2.7(2.6)$ & \\
\hline Total social support baseline mean (SD) & $82.2(15.6)$ & $75.9(18.9)$ & $79.1(14.8)$ & $80.4(16.1)$ & 0.40 \\
\hline
\end{tabular}


Table 3 Final hierarchical regression analysis of PTG $\times$ PTS interaction at 6-month follow-up on HRQoL and psychological distress at 24-month follow-up

$\beta$

Mental HRQoL (MCS)

Treatment status (off treatment)

Occupational status

$0.25 * *$

(not at school/job)

Social support

-0.20 *

Symptoms at 24-month follow-up

0.05

PTS at 6-month follow-up

PTG at 6-month follow-up

PTS $\times$ PTG

$F$

$R^{2}$

$-0.18 *$

$-0.42 * *$

$0.19 *$

$-0.07$

$9.26 * *$

0.42

Physical HRQoL (PCS)

Occupational status

(not at school/job)

Social support

Symptoms at 24-month follow-up

PTS at 6-month follow-up

PTG at 6-month follow-up

$\mathrm{PTS} \times \mathrm{PTG}$

F

$R^{2}$

$-0.11$

0.13

$-0.38 * *$

$-0.13$

0.04

0.11

$5.57 * *$

0.27

Psychological distress

Treatment status (off treatment)

Occupational status (not at school/job)

$-0.16^{*}$

0.09

$-0.13$

$0.35 * *$

$0.48 * *$

-0.14 *

$-0.12$

$17.14 * *$

0.56

on bivariate level. No relationship between PTG and physical HRQoL was found. Note that PTS also remained a significant predictor of mental HRQoL and psychological distress (Table 3).

\section{Buffering effect of PTG on link between PTS and HRQoL and psychological distress}

Results revealed no significant interaction effects between PTS and PTG on outcomes, indicating that buffering did not take place (Table 3).

\section{Discussion}

In this prospective follow-up study, we found that most AYAs with cancer experience some degree of PTG between 6 and 24 months after baseline assessment. In accordance with the literature, the most frequently scored PTGI items included a sense of enhanced life appreciation, reinforced interpersonal relationships, and personal strengthening, whereas finding new opportunities and spiritual change were the least endorsed areas of growth [25-27, 34, 35]. Our study adds to the current literature by showing that PTG is a dynamic process within individuals. Although no significant mean differences in PTG scores were found over time within the sample as a whole, we were able to identify four trajectories of individual change or stableness: high PTG, increasing PTG, decreasing PTG, and low PTG. Interestingly, the number of individuals who showed increasing PTG over time approximately matched those who showed decreasing PTG. A major goal for future work should be increasing the prevalence of increasing PTG profiles.

When looking at factors promoting or preventing PTG in different studies, our findings show that females, younger AYAs, and those who received chemotherapy more often were found in the high PTG group and least often in the low PTG group. Some researchers have suggested that women are, on average, more emotion-focused in their coping strategies and may therefore be more often able than men to derive a sense of positive meaning from the traumatic experience of cancer [10, 36]. Younger patients might be more aware of and motivated to conform to expectations to adopt a positive attitude in the face of cancer than are older patients [27, 37]. It may also be that a cancer diagnosis is more stressful and necessitates more developmental readjustment for younger AYAs [10], leading to more PTG. The other way around, the impact of cancer on younger AYAs is happening at a time when most of them still 
have support of their family/parents, they are just starting out on life, have not yet gained (financial) independence or are still reliant on parents, and do not have children or families of their own to worry about whilst those who are diagnosed as older AYAs may have all these responsibilities of having a job, family, and mortgage, which may potentially make a cancer diagnosis more life disrupting and may limit them to find benefit of their cancer experience. It could also be that older AYAs are more aware of what a cancer diagnosis may mean for them in terms of their health and their future. One might also hypothesize that receipt of more aversive treatments that produce greater life disruption (e.g., chemotherapy) might set the stage for greater PTG [10]; however, studies have demonstrated no association between chemotherapy and PTG [20, $26,35]$.

Findings to date regarding PTG following a cancer diagnosis have been quite inconsistent regarding relationships with well-being; PTG is sometimes shown to be positively related to adjustment to cancer, but sometimes inversely, and often with little relationship at all $[10,19]$. We found a direct positive predictive effect of PTG on mental HRQoL and a direct negative predictive effect on psychological distress. This suggests that PTG may reflect a cognitive adaptation process in response to a cancer diagnosis $[19,20]$, helping the AYAs to better mentally adjust to the cancer experience. PTG may provide a positive engagement with life, strengthening peoples' feelings of self-reliance and fostering social relationships [38], which may explain these direct relationships. On the other hand, PTG was not a predictor of physical HRQoL. It seems that this outcome is more influenced by the physical symptoms AYAs are currently coping with than by PTG. One explanation for the lack of this relationship could be that PTGI measures psychosocial areas of growth and not physical domains [39]. Future research could explore the predictive value of physical HRQoL for PTG.

Inconsistent with previous research identifying PTG as a buffer against the harmful effects of PTS [34, 40], PTG did not interact with PTS to predict these outcomes in our study. It could be hypothesized that PTS and PTG consist of different processes which may co-exist but are largely independent [13, $26,27,41]$. PTG reflects a coping process or resource that triggers the cognitive reconstruction of the individual's beliefs and strengths, while PTS is more an emotional process of intrusive thoughts, avoidance behavior, and heightened arousability with an aversive nature [42]. However, there is one study showing that re-experiencing trauma (a symptom of PTS) has a curvilinear relationship with two scales of the PTGI (new possibilities and personal strengths), showing that re-experiencing may also represent a cognitive process necessary to achieve personal growth for AYAs [13]. The authors state that opportunities to engage family, friends, cancer survivors, or health care professionals in frank discussions about fears, worries, or concerns may help AYAs re-experience cancer in a way that enhances their understanding of what happened to them and contributes to better mental well-being.

One potential implication of these findings is that helping AYAs with cancer who experience low or decreasing levels of PTG find meaning and benefits in the cancer experience may help improve their mental adjustment. This may be accomplished by encouraging the reappraisal of the situation (positive reframing) or the emotional disclosure of inner feelings and fears [43]. A written emotional disclosure intervention, whereby adult breast cancer survivors were randomized to write about positive thoughts and feelings related to their cancer, showed that those who wrote down positive feelings reported less physician visits for medical comorbidities and distress than survivors who wrote down facts of their experience [43]. In addition, two cognitive-behavioral interventions among adult cancer patients, that included elements of coping skills training, relaxation exercises, conflict resolution, and emotional expression, had beneficial effects on PTG [44, 45]. Another way to enhance PTG may be regular physical activity. The physiological benefit of physical activity may be stress reduction. Physical activity may also increase levels of social support for AYA cancer patients and serve as an active coping strategy to decrease levels of distress [15]. In addition, physical activity can improve AYA cancer patients' selfefficacy levels, whereby the experience of positive feelings may be enhanced. As PTG predicts mental well-being, interventions focused on improving PTG are likely beneficial in the early post-treatment period.

This study is limited by the fact that PTG trajectory group sizes were too small to conduct multivariate linear regression on outcomes. Therefore, we used mean-centered continuous PTG scores at 6-month follow-up to predict outcomes at 24month follow-up. Longitudinal mixed model analyses were not conducted because of the lack of HRQoL data at 6month follow-up. Furthermore, with regard to instrumentation, the current study relied upon generic instruments with limited evidence of validity and reliability in study samples consisting of persons under 18 years of age. In addition, we did not assess social desirability. Some cancer patients believe they should derive growth from their cancer experience and subsequently may report such growth regardless of their personal experience of positive change or benefit [41]. Similarly, some cancer patients know thinking and talking positively about their cancer experience will elicit positive attention from others [46]. Future research is needed to examine the meaning of PTG for AYAs in the broader contexts of their lives and the ways in which they process their experiences. For example, the four PTG trajectories could indicate that PTG functions as a coping style early after diagnosis but becomes real growth for some after a longer period of time [47].

In conclusion, this study shows that PTG is a dynamic process within the individual AYA cancer patient that predicts mental well-being outcomes but does not serve to buffer PTS. 
Psychosocial interventions should focus on both PTS and PTG in order to promote positive adjustment for AYAs diagnosed with cancer.

\section{Compliance with ethical standards}

Funding This study was supported by HopeLab Foundation, Redwood City, CA. Dr. Olga Husson (KUN2015-7527) is supported by a Social Psychology Fellowship from the Dutch Cancer Society. None of these funders had control over study design, data collection, data analysis, and reporting. The authors have full control of all primary data and will allow the journal to review the data if requested.

Conflict of interest The authors declare that they have no conflict of interest.

Ethical approval All procedures performed in studies involving human participants were in accordance with the ethical standards of the institutional and/or national research committee and with the 1964 Helsinki Declaration and its later amendments or comparable ethical standards.

Open Access This article is distributed under the terms of the Creative Commons Attribution-NonCommercial 4.0 International License (http:// creativecommons.org/licenses/by-nc/4.0/), which permits any noncommercial use, distribution, and reproduction in any medium, provided you give appropriate credit to the original author(s) and the source, provide a link to the Creative Commons license, and indicate if changes were made.

\section{References}

1. Adolescent and Young Adult Oncology Progress Review Group 2006. Closing the gap: research and care imperatives for adolescents and young adults with cancer. Bethesda, MD: National Institute of Health, National Cancer Institute, and Livestrong Young Ault Alliance, NIH Publication No. 06-6067

2. Haase JE, Phillips CR (2004) The adolescent/young adult experience. J Pediatr Oncol Nurs 21:145-149

3. Arnett JJ (2000) Emerging adulthood. A theory of development from the late teens through the twenties. Am Psychol 55:469-480

4. Thomas DM, Albritton KH, Ferrari A (2010) Adolescent and young adult oncology: an emerging field. J Clin Oncol 28:47814782

5. Zebrack BJ (2011) Psychological, social, and behavioral issues for young adults with cancer. Cancer 117:2289-2294

6. Smith AW, Bellizzi KM, Keegan TH et al (2013) Health-related quality of life of adolescent and young adult patients with cancer in the United States: the adolescent and young adult health outcomes and patient experience study. J Clin Oncol 31:2136-2145

7. Selye H (1956) The stress of life. McGraw-Hill, New York

8. Calhoun LG, Tedeschi RG (1999) Facilitating posttraumatic growth. A clinician's guide. Lawrence Erlbaum Associates, Mahwah

9. Calhoun LG, Tedeschi RG (2002) Posttraumatic growth: the positive lessons of loss. In: Neimeyer RA (ed) Meaning reconstruction and the experience of loss. American Psychological Association, Washington, DC, pp 157-172

10. Stanton AL, Bower JE, Low CA (2006) Posttraumatic growth after cancer. In: Calhoun LG, Tedeschi RG (eds) Handbook of posttraumatic growth: research and practice. Erlbaum Associates, Mahwah, New Jersey, pp 138-175
11. Wicks L, Mitchell A (2010) The adolescent cancer experience: loss of control and benefit finding. Eur J Cancer Care (Engl) 19:778785

12. Salsman JM, Garcia SF, Yanez B et al (2014) Physical, emotional, and social health differences between posttreatment young adults with cancer and matched healthy controls. Cancer 120:2247-2254

13. Zebrack B, Kwak M, Salsman J et al (2015) The relationship between posttraumatic stress and posttraumatic growth among adolescent and young adult (AYA) cancer patients. Psychooncology 24:162-168

14. Monteiro S, Torres A, Morgadinho R, Pereira A (2013) Psychosocial outcomes in young adults with cancer: emotional distress, quality of life and personal growth. Arch Psychiatr Nurs 27:299-305

15. Love C, Sabiston CM (2011) Exploring the links between physical activity and posttraumatic growth in young adult cancer survivors. Psychooncology 20:278-286

16. Seitz DC, Hagmann D, Besier T et al (2011) Life satisfaction in adult survivors of cancer during adolescence: what contributes to the latter satisfaction with life? Qual Life Res 20:225-236

17. Calhoun LG, Tedeschi RG (1995) Trauma and transformation: growing in the aftermath of suffering. SAGE Publications, Thousand Oaks, CA

18. Davis CG, Nolen-Hoeksema S, Larson J (1998) Making sense of loss and benefiting from the experience: two construals of meaning. J Pers Soc Psychol 75:561-574

19. Helgeson VS, Reynolds KA, Tomich PL (2006) A meta-analytic review of benefit finding and growth. J Consult Clin Psychol 74: $797-816$

20. Morrill EF, Brewer NT, O'Neill SC et al (2008) The interaction of post-traumatic growth and post-traumatic stress symptoms in predicting depressive symptoms and quality of life. Psychooncology 17:948-953

21. McCarthy MC, McNeil R, Drew S et al (2016) Psychological distress and posttraumatic stress symptoms in adolescents and young adults with cancer and their parents. J Adolesc Young Adult Oncol In press

22. Kwak M, Zebrack BJ, Meeske KA et al (2013) Prevalence and predictors of post-traumatic stress symptoms in adolescent and young adult cancer survivors: a 1-year follow-up study. Psychooncology 22:1798-1806

23. Meeske KA, Ruccione K, Globe DR, Stuber ML (2001) Posttraumatic stress, quality of life, and psychological distress in young adult survivors of childhood cancer. Oncol Nurs Forum 28: 481-489

24. Tedeschi RG, Calhoun LG (1996) The posttraumatic growth inventory: measuring the positive legacy of trauma. J Trauma Stress 9: 455-471

25. Gianinazzi ME, Rueegg CS, Vetsch J et al (2016) Cancer's positive flip side: posttraumatic growth after childhood cancer. Support Care Cancer 24:195-203

26. Cordova MJ, Giese-Davis J, Golant M, Kronenwetter C, Chang V, Spiegel D (2007) Breast cancer as trauma: posttraumatic stress and posttraumatic growth. J Clin Psychol Med Settings 14:308-319

27. Widows MR, Jacobsen PB, Booth-Jones M, Fields KK (2005) Predictors of posttraumatic growth following bone marrow transplantation for cancer. Health Psychol 24:266-273

28. Jansen L, Hoffmeister M, Chang-Claude J et al (2011) Benefit finding and post-traumatic growth in long-term colorectal cancer survivors: prevalence, determinants, and associations with quality of life. Br J Cancer 105:1158-1165

29. Sherbourne CD, Stewart AL (1991) The MOS social support survey. Soc Sci Med 32:705-714

30. Foa EB, Cashman L, Jaycox L, Perry K (1997) The validation of a self-report measure of posttraumatic stress disorder: the posttraumatic diagnostic scale. Psychol Assess 9:445-451 
31. Ware JE, Snow KK, Kosinski M et al (1993) SF-36 health survey: manual and interpretation guide. Boston, MA, The Health Institute, New England Medical Center

32. Derogatis LR (2001) Brief symptom inventory 18: administration, scoring, and procedures manual. Pearson, Bloomington

33. Howlader N, Noone AM, Krapcho M et al (2011) SEER cancer statistics review, 1975-2008. Bethesda, National Institutes of Health

34. Silva SM, Moreira HC, Canavarro MC (2012) Examining the links between perceived impact of breast cancer and psychosocial adjustment: the buffering role of posttraumatic growth. Psychooncology 21:409-418

35. Lelorain S, Bonnaud-Antignac A, Florin A (2010) Long term posttraumatic growth after breast cancer: prevalence, predictors and relationships with psychological health. J Clin Psychol Med Settings 17:14-22

36. Fife BL (1994) The conceptualization of meaning in illness. Soc Sci Med 38:309-316

37. Manne S, Ostroff J, Winkel G et al (2004) Posttraumatic growth after breast cancer: patient, partner, and couple perspectives. Psychosom Med 66:442-454

38. Pakenham KI (2005) Benefit finding in multiple sclerosis and associations with positive and negative outcomes. Health Psychol 24: $123-132$

39. Bellizzi KM, Smith AW, Reeve BB et al (2010) Posttraumatic growth and health-related quality of life in a racially diverse cohort of breast cancer survivors. J Health Psychol 15:615-626

40. Park CL, Chmielewski J, Blank TO (2010) Post-traumatic growth: finding positive meaning in cancer survivorship moderates the impact of intrusive thoughts on adjustment in younger adults. Psychooncology 19:1139-1147

41. Salsman JM, Segerstrom SC, Brechting EH et al (2009) Posttraumatic growth and PTSD symptomatology among colorectal cancer survivors: a 3-month longitudinal examination of cognitive processing. Psychooncology 18:30-41

42. Andrykowski MA, Cordova MJ, Studts JL, Miller TW (1998) Posttraumatic stress disorder after treatment for breast cancer: prevalence of diagnosis and use of the PTSD Checklist-Civilian Version (PCL-C) as a screening instrument. J Consult Clin Psychol 66:586590

43. Stanton AL, Danoff-Burg S, Sworowski LA et al (2002) Randomized, controlled trial of written emotional expression and benefit finding in breast cancer patients. J Clin Oncol 20:41604168

44. Antoni MH, Lehman JM, Kilbourn KM et al (2001) Cognitivebehavioral stress management intervention decreases the prevalence of depression and enhances benefit finding among women under treatment for early-stage breast cancer. Health Psychol 20: 20-32

45. Penedo FJ, Dahn JR, Molton I et al (2004) Cognitive-behavioral stress management improves stress-management skills and quality of life in men recovering from treatment of prostate carcinoma. Cancer 100:192-200

46. Wilkinson S, Kitzinger C (2000) Thinking differently about thinking positive: a discursive approach to cancer patients' talk. Soc Sci Med 50:797-811

47. Sawyer A, Ayers S, Field AP (2010) Posttraumatic growth and adjustment among individuals with cancer or HIV/AIDS: a metaanalysis. Clin Psychol Rev 30:436-447 